TAIWANESE JOURNAL OF MATHEMATICS

Vol. 13, No. 6B, pp. 2093-2103, December 2009

This paper is available online at http://www.tjm.nsysu.edu.tw/

\title{
EXACT NULL CONTROLLABILITY OF ABSTRACT SEMILINEAR FUNCTIONAL INTEGRODIFFERENTIAL STOCHASTIC EVOLUTION EQUATIONS IN HILBERT SPACES
}

\author{
J. Y. Park ${ }^{1}$ and P. Balasubramaniam ${ }^{2, *}$
}

\begin{abstract}
Sufficient conditions for exact null controllability of the semilinear integrodifferential stochastic evolution equations in Hilbert space are obtained. It is shown that the exact null controllability of the corresponding linear system with additive term implies the exact null controllability of the semilinear functional integrodifferential stochastic evolution equations. An application to stochastic partial integrodifferential equations is given.
\end{abstract}

\section{INTRODUCTION}

Semigroup theory gives a unified treatment of a wide class of stochastic parabolic, hyperbolic and functional differential equations, and much effort has been devoted to the study of controllability results for such evolution equations. In deterministic cases, fixed point techniques, among the other methods, are widely used as a tool for studying the controllability of nonlinear systems. Several authors have extended finite dimensional controllability results to infinite dimensional controllability results represented by integrodifferential evolution equations with bounded and unbounded linear operators in Banach spaces see the early survey by Balachandran and Dauer [2] and the references therein. Stochastic control theory is a stochastic generalization of classical control theory. Controllability of linear stochastic systems is a

Received January 11, 2006, accepted December 8, 2007.

Communicated by Yuan-Chung Sheu.

2000 Mathematics Subject Classification: 93E03, 93C40.

Key words and phrases: Exact null controllability, Integrodifferential equations, Semilinear stochastic evolution equation, Schauder fixed point theorem.

${ }^{1}$ The work was supported by grant no. KRF-2005-202-C00030 from the Basic Research Program of the Korea Research Foundation.

${ }^{2}$ The author work was supported by the KOSEF at the Pusan National University, South Korea during his visiting 2005-06 from Department of Mathematics, Gandhigram Rural Institute-Deemed University, Gandhigram- 624 302, Tamilnadu, India,

*Correspondence Author. 
well-known problem discussed in the literature $([1,8,11,14])$. Controllability of a linear stochastic system in Hilbert space recently has been extended by Balasubramaniam and Dauer[3] to the stochastic evolution equation with infinite delay. The controllability results in the infinite dimensional cases have been studied by several authors by the assumption that the semigroup $T(t), t>0$, associated with the linear convolution operator $L_{0}^{b} u=\int_{0}^{b} T(b-s) B u(s) d s$ has a bounded inverse operator $\left(L_{0}\right)^{-1}$ with values in $L^{2}(J, U) / \operatorname{ker}\left(L_{0}^{b}\right)$. The main objective of this paper (section 2 ) is to remove the bounded invertibility condition replacing it by the exact null controllability of the associated linear system with additive term in the stochastic settings based on the recent observation of [7]. Using this operator we transform the controllability problem into a fixed point theorem to show that the operator has a fixed point.

The purpose of this paper is to study the exact null controllability results of a class of abstract semilinear functional integrodifferential stochastic evolution equations of the form

$$
\begin{aligned}
d(x(t))= & {\left[A x(t)+B u(t)+F\left(t, x_{t}\right)\right] d t+\int_{0}^{t} G\left(s, x_{s}\right) d w(s), } \\
& \text { for a .e } t \in J=[0, b], \\
x_{0}(\theta)= & \phi(\theta), \quad \theta \in[-r, 0]
\end{aligned}
$$

where $A$ is the infinitesimal generator of a strongly continuous semigroup $T(t)$ in a real separable Hilbert space $\mathrm{H}$, the state $x(\cdot)$ takes its values in $H$ and the control function $u$ is given in $L^{2}(\mathrm{~J}, \mathrm{U})$, a Hilbert space of admissible control functions with $U$ as a separable Hilbert space. $B$ is a bounded linear operator from $U$ into $H$. Here $w$ is an $H$-valued Wiener process associated with a positive, nuclear covariance operator $Q, F$ is an $H$-valued map and $G$ is a $L(K, H)$-valued map both defined on $J \times C_{r}$ (where $K$ is a real separable Hilbert space and $L(K, H)$ is the space of all bounded, linear operators from $K$ to $H$, we write simply $L(H)$ if $H=K$.) and $\phi$ is an $C_{r}$-valued random variable independent of $w$ with finite second moment. Here $C_{r}=C([-r, 0], H)$ is a Banach space of all continuous functions $\phi:[-r, 0] \rightarrow H$ endowed with the norm $\|\phi\|=\sup \{\|\phi(\theta)\|:-r \leq \theta \leq 0\}$. Let $J_{1}=[-r, b]$, for $x(\cdot) \in C\left(J_{1}, H\right)$ we have $x_{t}(\cdot) \in C_{r}$ for $t \in J, x_{t}(\theta)=x(t+\theta)$ for $\theta \in[-r, 0]$. Our aim is to study the exact null controllability of the mild solution of the system (1) that is exact null controllability of the following system

$$
\begin{aligned}
x(t)= & T(t) \phi(0)+\int_{0}^{t} T(t-s)\left[B u(s)+F\left(s, x_{s}\right)+\int_{0}^{s} G\left(\tau, x_{\tau}\right) d w(\tau)\right] d s, \\
& \text { for a .et } \in J=[0, b], \\
x_{0}(\theta)= & \phi(\theta), \quad \theta \in[-r, 0]
\end{aligned}
$$


The integrodifferential equation considered here serves as an abstract formulation of partial integrodifferential equation which arise in various physical phenomena such as electromagnetic theory, population dynamics, and heat conduction in materials with memory (for details see $([4,9,10,14])$.

The outlay of the paper is as follows. In the following section, we give the necessary preliminaries, definitions and stated hypotheses for proving the controllability result. In section 3 , we deduce the main result to that of finding a fixed point of a solution map. Finally in section 4, an example is presented which illustrates the main theorem.

\section{Preliminaries}

For more details of this section, the reader may refer ([6], [13]) and the references therein. Throughout the paper, $(H,\|\cdot\|)$ and $\left(K,\|\cdot\|_{K}\right)$ denote real separable Hilbert spaces.

Let $(\Omega, \mathfrak{F}, P)$ be a complete probability space furnished with complete family of right continuous increasing sub $\sigma$-algebras $\left\{\mathfrak{F}_{t}, t \in J\right\}$ satisfying $\mathfrak{F}_{t} \subset \mathfrak{F}$. An $H$ valued random variable is an $\mathfrak{F}$-measurable function $x(t): \Omega \rightarrow H$ and a collection of random variables $S=\{x(t, w): \Omega \rightarrow H \mid t \in J\}$ is called a stochastic process. Usually we suppress the dependence on $w \in \Omega$ and write $x(t)$ instead of $x(t, w)$ and $x(t): J \rightarrow H$ in the place of $S$. Let $\beta_{n}(t)(n=1,2, \ldots)$ be a sequence of real-valued one-dimensional standard Brownian motions mutually independent over $(\Omega, \mathfrak{F}, P)$. Set $w(t)=\sum_{n=1}^{\infty} \sqrt{\lambda_{n}} \beta_{n}(t) \zeta_{n}, t \geq 0$, where $\lambda_{n} \geq 0,(\mathrm{n}=1,2$, ...) are nonnegative real numbers and $\left\{\zeta_{n}\right\}(\mathrm{n}=1,2, \ldots)$ is complete orthonormal basis in $K$. Let $Q \in L(K, K)$ be an operator defined by $Q \zeta_{n}=\lambda_{n} \zeta_{n}$ with finite $\operatorname{Tr}(Q)=\sum_{n=1}^{\infty} \lambda_{n}<\infty$, (Tr denotes the trace of the operator). Then the above $K$-valued stochastic process $w(t)$ is called a $Q$-Wiener process. We assume that $\mathfrak{F}_{t}=\sigma(w(s): 0 \leq s \leq t)$ is the $\sigma$-algebra generated by $w$ and $\mathfrak{F}_{T}=\mathfrak{F}$. Let $\varphi \in L(K, H)$ and define

$$
\|\varphi\|_{Q}^{2}=\operatorname{Tr}\left(\varphi Q \varphi^{*}\right)=\sum_{n=1}^{\infty}\left\|\sqrt{\lambda_{n}} \varphi \zeta_{n}\right\|^{2} .
$$

If $\|\varphi\|_{Q}<\infty$, then $\varphi$ is called a $Q$-Hilbert-Schmidt operator. Let $L_{Q}(K, H)$ denote the space of all $Q$-Hilbert-Schmidt operators $\varphi: K \rightarrow H$. The completion $L_{Q}(K, H)$ of $L(K, H)$ with respect to the topology induced by the norm $\|\cdot\|_{Q}$ where $\|\varphi\|_{Q}=\langle\langle\varphi, \varphi\rangle\rangle^{1 / 2}$ is a Hilbert space with the above norm topology.

Finally, let $C\left(J_{1}, L_{2}(\Omega ; H)\right)$ be the space of all continuous functions from $J_{1}$ into $L_{2}(\Omega ; H)$ satisfying the conditions $\sup _{t \in J_{1}} E\|x(t)\|^{2}<\infty, E$ stands for integration with respect to probability measure $[P]$. Then, define the subspace

$$
Y=C\left(J_{1}, H\right)=\left\{\xi \in C\left(J_{1}, L_{2}^{\mathfrak{F}}(\Omega, H)\right) \mid \xi \text { is } \mathfrak{F}_{t} \text {-adapted }\right\}
$$


denote the family of continuous $H$-valued stochastic processes $\left\{\xi(t): t \in J_{1}\right\}$ which are $\mathcal{F}_{t}$-measurable and have finite second moments, that is,

$$
\|\xi\|_{Y}=\sup _{t \in J_{1}}\left(E\|\xi(t)\|^{2}\right)^{1 / 2}<\infty .
$$

It is easy to verify that $Y$ furnished with the norm topology as defined above, is a Banach space.

Definition 2.1. The system (2) is said to be exact null controllable on the interval $J$, if for every $\phi$ and preassigned time $b$ there exists a stochastic control $u \in L_{2}(J, U)$ such that the solution $x(\cdot)$ satisfies $x(b)=0$.

Define $L_{0}^{b} u=\int_{0}^{b} T(b-s) B u(s) d s: L_{2}(J, U) \rightarrow H$,

$N_{0}^{b}(z, f)=T(b) z+\int_{0}^{b} T(b-s) f(s) d s: H \times L_{2}(J, U) \rightarrow H$,

where $f(s)=F(s)+\int_{0}^{s} G(\tau) d w(\tau)$

Consider the linear system

$$
\begin{aligned}
\frac{d}{d t}(z(t)) & =A z(t)+B u(t)+f(t), \text { for a .e } t \in J=[0, b], \\
z(0) & =z_{0}, \quad \theta \in[-r, 0]
\end{aligned}
$$

associated with the system (1).

Definition 2.2. The system (3) is said to be exactly null controllable on $J$ if

$$
\operatorname{Im} L_{0}^{b} \supset \operatorname{Im} N_{0}^{b} \text {. }
$$

Remark 2.1. It is known that, see [5], system (3) is exactly null controllable if and only if there exists $\gamma>0$ such that

$$
\left\|\left(L_{0}^{b}\right)^{*}\right\|^{2} \geq \gamma\left\|\left(N_{0}^{b}\right)^{*} z\right\|^{2} \text { for all } z \in H .
$$

The following lemma is crucial in the part of our main result and has been proven in [7].

Lemma 2.1. Suppose that the system (3) is exactly null controllable on J. Then the linear operator $W:\left(L_{0}\right)^{-1} N_{0}^{b}: H \times L_{2}(J, H) \rightarrow L_{2}(J, U)$ is bounded and the control

$$
\begin{aligned}
u(t) & =-\left(L_{0}\right)^{-1}\left[T(b) z_{0}+\int_{0}^{b} T(b-s) F(s) d s+\int_{0}^{b} T(b-s) G(s) d w(s)\right] \\
& =-W\left(z_{0}, F, G\right)
\end{aligned}
$$

transfers the system (3) from $z_{0}$ to 0 , where $L_{0}$ is the restriction of $L_{0}^{b}$ to $\left[k e r L_{0}^{b}\right]^{\perp}$, $F \in L_{2}(J, H)$ and $G \in L_{2}(J, L(K, H))$. 
For the proof of the main result in section 3, we will use the following hypotheses:

$(C 1)$ The compact semigroup $T(t)$ on the separable Hilbert space $\mathrm{H}$, there exists constant $M_{T}$ such that

$$
\|T(t)\| \leq M_{T}, t \geq 0,
$$

$(C 2)$ The linear system (3) is exactly null controllable on $\mathbf{J}$.

(C3) $w$ is a $H$-valued Wiener process defined on a Hilbert space $K$.

(C4) The functions $F: J \times C_{r} \rightarrow H$ and $G: J \times C_{r} \rightarrow L(K, H)$ are continuous, for each $x \in H$ the functions $F(\cdot, x): J \rightarrow H$ and $G(\cdot, x): J \rightarrow L\left(K_{Q}, H\right)$ are strongly $\mathcal{F}_{t}$-measurable and there exists functions $\lambda(\cdot) \in L_{1}\left(J, R^{+}\right)$and $g(\cdot) \in L_{1}\left(C_{r}, R^{+}\right)$be such that for a.a. $(t, \phi) \in J \times C_{r}$

$$
E\|F(t, \phi)\| \bigvee E\|G(t, \phi)\|_{Q} \leq \lambda(t) g(\phi) .
$$

(C5) Next, for convenience, let us introduce the following assumptions

$$
\begin{aligned}
\|\lambda\|_{2}= & \left(\int_{0}^{b}\|\lambda(s)\|^{2} d s\right)^{1 / 2} \\
k= & \max \left\{1, M_{W}, M_{B} M_{T} \sqrt{b}\right\}, \quad \text { where } M_{W}=\|W\| \text { and } M_{B}=\|B\| \\
c= & \max \left\{c_{1}, c_{2}\right\}, \quad \text { where } c_{1}=2 k M_{W} \sqrt{b}\left(\|\lambda\|+\sqrt{\operatorname{Tr}(Q)}\|\lambda\|_{2}\right) \\
& \text { and } c_{2}=2 M_{T} b\left(\|\lambda\|+\sqrt{\operatorname{Tr}(Q)}\|\lambda\|_{2}\right) \\
d= & \max \left\{d_{1}, d_{2}\right\}, \quad \text { where } d_{1}=2 k M_{W}\|\phi(0)\| \text { and } d_{2}=2 M_{T}\|\phi(0)\| .
\end{aligned}
$$

(C5) $\lim \sup _{r \rightarrow \infty}(r-\operatorname{csup}\{g(\phi):\|\phi\| \leq r\})=\infty$.

\section{Controllability Results}

Theorem 3.1. Suppose the conditions $(C 1)-(C 6)$ are satisfied. Then the system (2) is exactly null controllable on J.

Proof. Using the hypothesis $(\mathrm{C} 2)$ for an arbitrary $x(\cdot)$ define the operator $\Phi$ on $Y$ as follows

$$
\text { (4) }(\Phi x)(t)=\left\{\begin{array}{l}
\phi(t), \text { if } \quad t \in[-r, 0], \\
T(t) \phi(0)+\int_{0}^{t} T(t-s)(-B W(\phi(0), F, G)) d s \\
\quad+\int_{0}^{t} T(t-s) F\left(s, x_{s}\right) d s \\
\quad+\int_{0}^{t} T(t-s)\left(\int_{0}^{s} G\left(\tau, x_{\tau}\right) d w(\tau)\right) d s, \text { for a. e. } t \in J,
\end{array}\right.
$$


where

$W(\phi(0), F, G)=\left(L_{0}\right)^{-1}\left[T(b) \phi(0)+\int_{0}^{b} T(b-s)\left(F\left(s, x_{s}\right)+\int_{0}^{s} G\left(\tau, x_{\tau}\right) d w(\tau)\right) d s\right]$.

It will be shown that the operator $\Phi$ from $Y$ into itself has a fixed point. On the Banach space $Y$ introduce a set

$$
Y_{r}=\left\{x(\cdot) \in Y: x(t)=\phi(t), t \in[-r, 0],\|x(t)\|_{Y} \leq r \text { for all } t \in J_{1}\right\}
$$

where $r$ is the positive constant. Let

$$
\psi(r)=\sup \{g(\phi):\|\phi\| \leq r\} .
$$

By the hypothesis (C6), there exists $r>0$ such that

$$
d+c \psi(r) \leq r .
$$

The proof will be given in several steps.

Step 1. The control $u(\cdot)=-W(\phi(0), F, G)$ is bounded on $Y_{r}$. Indeed,

$$
\begin{aligned}
E\|u\| \leq & \left(\int_{0}^{b} E\|W(\phi(0), F, G)(s)\|^{2} d s\right)^{1 / 2} \\
\leq & M_{W}\left[E\|\phi(0)\|+\left(\int_{0}^{t} E\left\|F\left(s, x_{s}\right)\right\|^{2} d s\right)^{1 / 2}\right. \\
& \left.+\left(\int_{0}^{b}\left(\int_{0}^{s} E\left\|G\left(\tau, x_{\tau}\right) d w(\tau)\right\|\right)^{2} d s\right)^{1 / 2}\right] .
\end{aligned}
$$

That is

$$
\begin{aligned}
\|u\| & \leq M_{W}\left[\|\phi(0)\|+\sqrt{b}\|\lambda\| \psi(r)+\sqrt{\operatorname{Tr}(Q)} \sqrt{b}\left(\int_{0}^{s}\|\lambda(\tau)\|^{2} d \tau\right)^{1 / 2} \psi(r)\right] \\
& \leq M_{W}\left[\|\phi(0)\|+\sqrt{b}\left(\|\lambda\|+\sqrt{\operatorname{Tr}(Q)}\|\lambda\|_{2}\right) \psi(r)\right] .
\end{aligned}
$$

Step 2. There exists $r>0$ such $\Phi$ sends $Y_{r}$ into itself, $\Phi: Y_{r} \rightarrow Y_{r}$. If $x(\cdot) \in Y_{r}$, from (4) and (5) for $t \in J$, we have

$$
\begin{aligned}
E\|(\Phi x)(t)\| \leq & M_{T} E\|\phi(0)\|+M_{T} M_{B} \sqrt{b}\left(\int_{0}^{b} E\|W(\phi(0), F, G)(s)\|^{2} d s\right)^{1 / 2} \\
& +M_{T} \sqrt{b}\left(\int_{0}^{t} E\left\|F\left(s, x_{s}\right)\right\|^{2} d s\right)^{1 / 2} \\
& +M_{T} \sqrt{b}\left(\int_{0}^{t} \int_{0}^{s} E\left\|G\left(\tau, x_{\tau}\right) d w(\tau)\right\|^{2} d s\right)^{1 / 2}
\end{aligned}
$$




$$
\begin{aligned}
& \|(\Phi x)(t)\| \\
\leq & M_{T}\|\phi(0)\|+M_{T} M_{B} \sqrt{b} M_{W}\left[\|\phi(0)\|+\sqrt{b}\left(\|\lambda\|+\sqrt{\operatorname{Tr}(Q)}\|\lambda\|_{2}\right) \psi(r)\right] \\
& +M_{T} b\left(\|\lambda\|+\sqrt{\operatorname{Tr}(Q)}\|\lambda\|_{2}\right) \psi(r) \\
\leq & \frac{d}{2}+\frac{d}{2}+k M_{W}\left[\sqrt{b}\left(\|\lambda\|+\sqrt{\operatorname{Tr}(Q)}\|\lambda\|_{2}\right) \psi(r)\right] \\
& +M_{T} b\left(\|\lambda\|+\sqrt{\operatorname{Tr}(Q)}\|\lambda\|_{2}\right) \psi(r) \\
\leq & \frac{1}{2}(d+c \psi(r))+\frac{1}{2}(d+c \psi(r)) \\
\leq & r .
\end{aligned}
$$

Hence, $\Phi$ maps $Y_{r}$ into itself.

Step 3. The operator $\Phi$ maps $Y_{r}$ into equicontinuous set of $Y_{r}$. Let $0<t_{1}<$ $t_{2} \leq b$. For each $x \in Y_{r}$, we have

$$
\begin{aligned}
z\left(t_{1}\right)-z\left(t_{2}\right) \leq & {\left[T\left(t_{1}\right)-T\left(t_{2}\right)\right] \phi(0)-\int_{t_{1}}^{t_{2}} T\left(t_{2}-s\right) B W(\phi(0), F, G)(s) d s } \\
& +\int_{0}^{t_{1}}\left[T\left(t_{1}-s\right)-T\left(t_{2}-s\right)\right] B W(\phi(0), F, G)(s) d s \\
& -\int_{t_{1}}^{t_{2}} T\left(t_{2}-s\right)\left[F\left(s, x_{s}\right)+\int_{0}^{s} G\left(\tau, x_{\tau}\right) d w(\tau)\right] d s \\
& +\int_{0}^{t_{1}}\left[T\left(t_{1}-s\right)-T\left(t_{2}-s\right)\right]\left[F\left(s, x_{s}\right)+\int_{0}^{s} G\left(\tau, x_{\tau}\right) d w(\tau)\right] d s .
\end{aligned}
$$

Therefore,

(6)

$$
\begin{aligned}
& \left\|z\left(t_{1}\right)-z\left(t_{2}\right)\right\| \\
\leq & \left\|T\left(t_{1}\right)-T\left(t_{2}\right)\right\|\|\phi(0)\|+M_{T} M_{B} \int_{t_{1}}^{t_{2}}\|W(\phi(0), F, G)(s)\| d s \\
& +M_{B} \int_{0}^{t_{1}}\left\|T\left(t_{1}-s\right)-T\left(t_{2}-s\right)\right\|\|W(\phi(0), F, G)(s)\| d s \\
& +M_{T} \int_{t_{1}}^{t_{2}}\left(\lambda(s)+\int_{0}^{s} \lambda(\tau) d w(\tau)\right) d s \psi(r) \\
& +\int_{0}^{t_{1}}\left\|T\left(t_{2}-s\right)-T\left(t_{1}-s\right)\right\|\left(\lambda(s)+\int_{0}^{s} \lambda(\tau) d w(\tau)\right) d s \psi(r) \\
= & I_{1}+I_{2}+I_{3}+I_{4}+I_{5} .
\end{aligned}
$$

Since by (5) the control $u$ is bounded, the right hand side (6) does not depend on particular choices of $x(\cdot)$. It is clear that $I_{2} \rightarrow 0$ and $I_{4} \rightarrow 0$ as $t_{1}-t_{2} \rightarrow 0$. 
Since the semigroup $T(\cdot)$ is compact, $\left\|T\left(t_{2}-s\right)-T\left(t_{1}-s\right)\right\| \rightarrow 0$ as $t_{1}-t_{2} \rightarrow 0$ for arbitrary $t, s$ such that $t-s>0$. Then $I_{1} \rightarrow 0$ and by Lebesque's dominated convergence theorem, $I_{3} \rightarrow 0$ and $I_{5} \rightarrow 0$ as $t_{1}-t_{2} \rightarrow 0$. As $t_{1}-t_{2} \rightarrow 0$, the right-hand side of (6) tends to zero. The equicontinuity for the cases $t_{1}<t_{2} \leq 0$ and $t_{1} \leq 0 \leq t_{2}$ follows from the uniform continuity of $\phi$ on the interval $[-r, 0]$.

Step 4. For arbitrary $t \in J$ the set

$$
V(t)=\left\{(\Phi x)(t): x(\cdot) \in Y_{r}\right\}
$$

is relatively compact. In fact, the case where $t=0$ is trivial, since $V(0)=\{\phi(0)\}$. So, let $t, 0<t \leq b$, be a fixed and let $\eta$ be a real numbers satisfying $0<\eta<t$. For every $x(\cdot) \in Y_{r}$ define

$$
\begin{aligned}
\left(\Phi_{\eta} x\right)(t)= & T(t) \phi(0)+T(\eta) \int_{0}^{t-\eta} T(t-s-\eta)(-B W(\phi(0), F, G) \\
& \left.+F\left(s, x_{s}\right)+\int_{0}^{s} G\left(\tau, x_{\tau}\right) d w(\tau)\right) d s .
\end{aligned}
$$

Since $T(\eta)$ is compact, the set

$$
V_{\eta}(t)=\left\{\left(\Phi_{\eta} x\right)(t): x(\cdot) \in Y_{r}\right\}
$$

is relatively compact set in $H$ for every $\eta, 0<\eta<t$. On the otherhand, for every $x(\cdot) \in Y_{r}$ by (6) we have

$$
\begin{aligned}
& \left\|(\Phi x)(t)-\left(\Phi_{\eta} x\right)(t)\right\| \\
= & \| \int_{t-\eta}^{t} T(t-s)[B W(\phi(0), F, G)(s) \\
& \left.+F\left(s, x_{s}\right)+\int_{0}^{s} G\left(\tau, x_{\tau}\right) d w(\tau)\right] d s \| \\
\leq & \left(\int_{t-\eta}^{t}\|T(t-s)\|^{2}\|B\|^{2} d s\right)^{1 / 2}\left(\int_{0}^{b}\|W(\phi(0), F, G)(s)\|^{2} d s\right)^{1 / 2} \\
& +M_{T}\|\lambda\| \psi(r) \eta+M_{T} \sqrt{\operatorname{Tr}(Q)}\|\lambda\|_{2} \psi(r) \eta \\
\leq & M_{T} M_{B} M_{W}\left[\|\phi(0)\|+\sqrt{b}\left(\|\lambda\|+\sqrt{\operatorname{Tr}(Q)}\|\lambda\|_{2}\right) \psi(r)\right] \\
& +M_{T} \eta\left(\|\lambda\|+\sqrt{\operatorname{Tr}(Q)}\|\lambda\|_{2}\right) \psi(r) \\
\leq & \varepsilon .
\end{aligned}
$$

Therefore there are relatively compact sets arbitrarily close to the set $V(t)$. Hence, for each $t \in J$, the set $V(t)$ is relatively compact in $H$ (see Pazy [12]). 
From the steps 2-4 and by the Ascoli-Arzela theorem, one can conclude that $\Phi$ is compact. On the other hand, it is easy to see tat $\Phi$ is continuous on $Y_{r}$. Hence, $\Phi$ is a compact continuous operator on $Y_{r}$. From the Schauder fixed point theorem $\Phi$ has a fixed point. Thus, the system (1) is exactly null controllable on $J$.

\section{Application}

The results from Section 3 is illustrated by showing its applicability to a semilinear partial integrodifferential equation.

Consider the partial differential system of the form

$$
\begin{aligned}
x_{t}(t, \theta)= & x_{\theta \theta}(t, \theta)+u(t, \theta)+F(s, x(s-h, \theta)) \\
& +\int_{0}^{t} G(s, x(s-h, \theta) d w(s), 0<x<1, t \in J, \\
x_{\theta}(t, 0)= & x_{\theta}(t, 1)=0, \quad t \in J \\
x(t, \theta)= & \phi(t, \theta), \quad x \in[-h, 0] .
\end{aligned}
$$

where $\phi$ is continuous, $u \in L_{2}(0, b)$ and $H=L_{2}(0,1)$. Also $F: R \times R \rightarrow R$ and $G: R \times R \rightarrow L(R)$ are continuous. Let $A: H \rightarrow H$ be operator defined by $A z=\frac{d^{2} z}{d \theta^{2}}$ with domain

$D(A)=\left\{z \in H: z, \frac{d z}{d \theta}\right.$ are absolutely continuous, $\left.z_{\theta \theta} \in H, \frac{d z}{d \theta}(0)=\frac{d z}{d \theta}(1)=0\right\}$.

It is known that $A$ is closed and $A$ has eigenvalues $\lambda_{n}=-n^{2} \pi^{2}, n \geq 0$, and the corresponding eigenvectors $e_{n}(\theta)=\sqrt{2} \cos (n \pi \theta)$ for $n \geq 1, e_{0}=1$, form an orthonormal basis for $L_{2}(0,1)$. Further, it is known that $A$ generates a compact semigroup $T(t), t>0$ in $\mathrm{H}$ and is given by

$$
\begin{aligned}
T(t) z & =(z, 1)+\sum_{n=1}^{\infty} e^{-n^{2} \pi^{2} t}\left(z, e_{n}\right) e_{n} \\
& =\int_{0}^{1} z(\alpha) d \alpha+\sum_{n=1}^{\infty}\left(2 e^{-n^{2} \pi^{2} t} \cos (n \pi \theta) \int_{0}^{1} \cos (n \pi \alpha) z(\alpha) d \alpha, z \in H,\right.
\end{aligned}
$$

and it is self-adjoint. If $u \in L_{2}(J, H)$, then $B=I, B^{*}=I$ and consequently by Remark 2.1, the condition for exact null controllability of the linear system with additive terms $F, G \in L_{2}(J, H)$,

$$
\begin{aligned}
& x_{t}(t, \theta)=x_{\theta \theta}(t, \theta)+u(t, \theta)+F(t, \theta)+\int_{0}^{t} G(t, \theta) d w(t), \\
& x_{\theta}(t, 0)=x_{\theta}(t, 1)=0, \quad t \in J \\
& x(t, \theta)=\phi(t, \theta), \quad x \in[-h, 0] .
\end{aligned}
$$


is the existence of a $\gamma>0$ such that

$$
\int_{0}^{b}\left\|B^{*} T^{*}(b-s) z\right\|^{2} d s \geq \gamma\left(\left\|T^{*}(b) z\right\|^{2}+\int_{0}^{b}\left\|T^{*}(b-s) z\right\|^{2} d s\right)
$$

or equivalently

$$
\int_{0}^{b}\left\|T^{*}(b-s) z\right\|^{2} d s \geq \gamma\left(\|T(b) z\|^{2}+\int_{0}^{b}\|T(b-s) z\|^{2} d s\right) .
$$

In [5], it is shown that the linear system (8) with $f=0$ is exactly null controllable if

$$
\int_{0}^{b}\|T(b-s) z\|^{2} d s \geq b\|T(b) z\|^{2}
$$

From here it follows that

$$
\int_{0}^{b}\|T(b-s) z\|^{2} d s \geq \frac{b}{1+b}\left(\|T(b) z\|^{2}+\int_{0}^{b}\|T(b-s) z\|^{2} d s\right) .
$$

Thus by Remark 2.1, the linear system (8) is exactly null controllable.

We assume that the nonlinear operators $F: J \times H \rightarrow H$ and $G: J \times H \rightarrow$ $L(K, H)$ are continuous and there is a constant $0<\gamma<1$ and a function $\alpha \in L_{2}(J)$ such that

$$
\|F(s, z)\| \bigvee\|G(s, z)\| \leq \alpha(s)\|z\|^{\gamma}
$$

for all $(s, z) \in J \times H$. So the conditions (C4) and (C6) are satisfied. Further, all the conditions stated in the above Theorem 3.1 are satisfied. Hence the system (7) is exactly null controllable on $J$.

\section{REFERENCES}

1. K. J. Astrom, Introduction to Stochastic Control Theory, Academic Press, New York, 1970.

2. K. Balachandran and J. P. Dauer, Controllability of nonlinear systems in Banach spaces: a survey, J. Optimiz. Theory Appl., 115 (2002), 7-28.

3. P. Balasubramaniam and J. P. Dauer, Controllability of semilinear stochastic evolution equations with nonlocal conditions, Int. J. Pure Appl. Math., 19 (2005), 281-296.

4. A. T. Bharucha-reid, Random Integral Equations, Academic Press, New York, 1982.

5. R. F. Curtain and H. Zwart, An Iintroduction to Infinite Dimensional Linear Systems Theory, Springer-Verlag, New York, 1995. 
6. G. Da Prato and J. Zabczyk, Stochastic Equations in Infinite Dimensions, Cambridge University Press, Cambridge, 1992.

7. J. P. Dauer and N. I. Mahmudov, Exact null Controllability of semilinear integrodifferential systems in Hilbert spaces, J. Math. Anal. Appl., 299 (2004), 322-332.

8. M. Ehrhard and W. Kliemann, Controllability of stochastic linear systems, System Control Lett., 2 (1982), 145-153.

9. W. Grecksch and C. Tudor, Stochastic Evolution Equations: A Hilbert Space Approach, Akademic Verlag, Berlin, 1995.

10. D. N. Keck and M. A. McKibben, Functional integro-differential stochastic evolution equations in Hilbert space, Journal of Applied Math. Sto. Anal., 16 (2003), 127-147.

11. N. I. Mahmudov, Controllability of linear stochastic systems in Hilbert spaces, $J$. Math. Anal. Appl., 259 (2001), 64-82.

12. A. Pazy, Semigroups of Linear Operators and Applications to Partial Differential Equations, Springer-Verlag, New York, 1983.

13. T. Taniguchi, K. Liu and A. Truman, Existence, uniqueness and asymptotic behavior of mild solutions to stochastic functional differential equations in Hilbert spaces, $J$. Differ. Equations, 181 (2002), 72-91.

14. J. Zabczyk, Controllability of stochastic linear systems, Systems Control Letters, 1 (1991), 25-31.

J. Y. Park and P. Balasubramaniam

Department of Mathematics,

Pusan National University,

Pusan, 609-735,

Korea

E-mail: jyepark@pusan.ac.kr pbalgri@pusan.ac.kr 